\title{
Competitividad para la internacionalización, frente a los atributos de innovación en Colombia- Caso Bucaramanga y su Área Metropolitana
}

\author{
Gladys Mireya Valero ${ }^{a}$, Manuel Rodenes ${ }^{b}$, Gladys Elena Rueda ${ }^{c}$ \\ aUniversidad Potificia Bolivariana, Bucaramanga, Colombia, gladys.valero@upb.edu.co \\ bUniversidad Politècnica de València, España, mrodenes2@gmail.com \\ cUniversidad Pontificia Bolivariana, Bucaramanga, Colombia, gladys.rueda@upb.edu.co
}

\begin{abstract}
Resumen
Favorecer la participación de las empresas colombianas en los mercados mundiales, como una estrategia internacional, se constituye en una necesidad importante, para Colombia y para las ciudades y regiones que la conforman. A través de una revisión de la literatura se consideran diversos autores que han efectuado aportes importanes de la innovación, elemento fundamental de la competitividad para la internacionalización. Asi mismo se toma una muestra de 63 empresas, a las que se les aplica el Coeficiente de Alfa de Cronbach para observar la importancia de la innovación en el trabajo, y poder identificar, en dicha revisión teórica y en la pertinencia de los resultados del coeficiente, si la innovación favorece la competitividad en las exportaciones de las empresas de Bucaramanga y su Area Metropolitana.
\end{abstract}

Palabras clave: Competitividad, Innovaciòn, Internacionalizaciòn, Empresas 
Competitividad para la internacionalizacion, frente a los atributos de innovacion en ColombiaCaso Bucaramanga y su Área Metropolitana

\section{Introducción}

En cualquier economía global, el proceso de internacionalización y la apertura de mercados son temas de especial importancia, para Colombia, este aspecto fortalece su importancia desde 1991, cuando se inserta en la economía internacional de manera más agresiva y menos tímida; a partir de ese año, el proceso de internacionalización es impulsado por la disposición de los gobiernos hacia los mercados mundiales, haciéndose evidente en los planes de desarrollo que Colombia ha adelantado desde ese año y han favorecido una creciente proyección exterior del tejido empresarial.

Actualmente, el número de empresas colombianas presentes en los mercados mundiales de bienes y servicios, ha aumentado progresivamente. Al tiempo, la vertiente internacional ha pasado a formar parte relevante de la estrategia de las empresas. No obstante, la presencia colombiana en el exterior aún está por debajo de su capacidad potencial, lo que implica continuar con esfuerzos que permitan ampliar la incursión de las empresas exportadoras en el mercado global y consoliden dicha perspectiva internacional.

Bajo este esquema y particularmente para el caso "Bucaramanga y su Area Metropolitan, se puede afirmar que las empresas conservan el mismo comportamiento; entonces a traves de la revisión teorica y la aplicación de un instrumento a las empresas exportadoras de la región, se busca identificar la importancia que la innovación representa en el proceso de internacionalización de las empresas; con el fin de identificar las subvariables que hacen de la competitividad a través de la inovaciòn, una òptima estrategia, para el mercado internacional en la región.

\section{Revisión de la Literatura}

De acuerdo al propósito de la presente revisión, se procede entonces a plantear un formato de modelo, que considera la innovación como la estrategia competitiva de internacionalización, para ello se inicia la revision teòrica de cada uno de las posibles subvariables, con el fin de ilustrar la importancia de las mismas dentro de cualquier estrategia competitiva en la inserción internacional (exportaciones) de las empresas 


\subsection{Innovación}

Ingresar al mercado internacional, implica hacerlo de manera competitiva y para ello se debe tener en cuenta el concepto de innovación. A lo largo del tiempo el concepto de innovación ha ido evolucionando considerablemente, en la siguiente tabla se encuentran algunos de los conceptos:

Tabla 1. Revisiòn Literatura Innovaciòn

\begin{tabular}{|c|c|c|}
\hline Autor & Conceptos & Planteamiento \\
\hline $\begin{array}{l}\text { Shumpeter } \\
\text { (1934) }\end{array}$ & $\begin{array}{l}\text { La teoría del Desarrollo } \\
\text { económico }\end{array}$ & $\begin{array}{l}\text { El desarrollo de las economías depende del nivel de innovación } \\
\text { proyectada en el PIB }\end{array}$ \\
\hline $\begin{array}{l}\text { Friedman } \\
\text { (1974) }\end{array}$ & La libre competencia & La integración entre tecnología e inventos genera la innovación \\
\hline $\begin{array}{l}\text { Drucker } \\
\text { (1988) }\end{array}$ & Gerencia del siglo XXI & La empresa innova cuando asigna recursos para ello \\
\hline $\begin{array}{l}\text { Porter } \\
\text { (1990) }\end{array}$ & Ventajas Competitivas & La innovación es el elemento clave que explica la competitividad \\
\hline $\begin{array}{l}\text { Fajnzylber } \\
\text { (1988) }\end{array}$ & Progreso Tecnológico & $\begin{array}{l}\text { La competitividad autèntica se obtiene cuando se logra incorporar } \\
\text { el progreso tecnológico de los bienes a partir de la innovación la } \\
\text { cual se logra a partir de la investigación y el desarrollo (I+D) }\end{array}$ \\
\hline $\begin{array}{l}\text { Bradley } \\
\text { (1997) }\end{array}$ & $\begin{array}{l}\text { Conocimiento } \\
\text { determinante de la } \\
\text { competitividad }\end{array}$ & $\begin{array}{l}\text { La innovación, el Capital tecnológico y el Capital intelectual } \\
\text { transforman los tangibles e intangibles en riqueza para las } \\
\text { empresas }\end{array}$ \\
\hline
\end{tabular}

Fuente: (Valero, Rodenes, \& Rueda, Effects of of the use of competitiveness as a strategy on exporting companies: Status of the issue, 2018)

Como se puede apreciar en todos los planteamientos anteriormente revisados, la innovación es plataforma fundamental en el proceso de internacionalización (exportaciones); pues tiene una relación positiva, que sumada a la productividad y a la inversión en Investigación y Desarrollo aportan a un mejor PIB (Simon Gaviria Muñoz, 2016), del país, aspecto fundamental en las exportaciones competitivas que se puedan consolidar.

En términos generales y de acuerdo a la consulta de la Metodología CIDEM-Maloka desarrollada por la Universidad del Rosario, (Universidad del Rosario, 2009), importante en este tema, se presenta a continuación los aspectos que se revisan para definir la Capacidad de exportación en Colombia ellos son: 
Competitividad para la internacionalizacion, frente a los atributos de innovacion en ColombiaCaso Bucaramanga y su Área Metropolitana

Atributos intrínsecos y de producción: se tiene ventaja competitiva o innovadora en materias primas, Se tiene ventaja competitiva o innovadora en diseño, Se tiene ventaja competitiva o innovadora en proceso de producción, Se tiene ventaja competitiva o innovadora en calidad, El producto tiene tramitados sus criterios de origen ante el Ministerio de Comercio Industria y Turismo, El producto está sujeto a controles por autoridades nacionales, El producto se identifica por algún código o referencia, Existe suficiente oferta de materias primas e insumos que permitan cumplir con entregas oportuna, Comparado con productos en el exterior el producto se debe adecuar, Ciclo de vida del producto, La empresa tiene identificado el nivel de competitividad del producto seleccionado para los mercados internacionales, El producto es un producto único en el mercado, El producto cumple con normas ambientales.

Atributos externos: se tiene ventaja competitiva o innovadora en empaques, Las etiquetas cumplen con requerimientos internacionales, Las cajas de cartón utilizadas como embalaje cuentan con la identificación de la empresa, Se tiene identificado el embalaje adecuado para el mercado internacional, El producto requiere de pictogramas para su manipulación, Existe alguna presentación en medios visuales, Se ajusta el empaque a las necesidades internacionales.

Atributos Intangibles: el producto cuenta con política de manejo de garantía, Se cuenta físicamente con un manual de uso y almacenamiento, La marca del producto se encuentra registrada ante la superintendencia de industria y comercio, El producto se encuentra

patentado ante alguna entidad, el manual se encuentra en idioma español-inglés, cuenta con ficha técnica.

Otros aspectos: cuenta la empresa con capacidad de producción para el mercado internacional. (Relacionada con el producto seleccionado), Se poseen los recursos técnicos, y profesionales y financieros necesarios para su comercialización, mantenimiento de la imagen de la empresa, La ficha técnica identifica los costos directos e indirectos del proceso productivo, crecimiento de las ventas, Esta establecido el costo de producción, Se ha identificado su partida arancelaria, El producto cuenta con preferencias arancelarias.

Como se puede apreciar la innovación es aspecto fundamental considerado en la metodología y permite, de acuerdo al planteamiento, ingresar de manera óptima al mercado internacional. Es de aclarar que dichos aspectos se ajustan dependiendo de la empresa, el sector y algunas particulares en el momento de definir la capacidad exportadora. 


\subsection{Competitividad}

En cuanto al concepto de competitividad se encuentra que históricamente, está ligado al comercio como actividad económica, en el siguiente cuadro se puede apreciar la evolución temporal del concepto:

Tabla 2. Revision literatura Competitividad

\begin{tabular}{|c|c|c|}
\hline Evolución histórica & Fuente & Aporte \\
\hline $\begin{array}{l}\text { 1.776 Los economistas clásicos } \\
\text { identificado los cuatro factores: } \\
\text { tierra, capital, recursos naturales } \\
\text { y fuerza laboral }\end{array}$ & $\begin{array}{l}\text { véase Adam Smith (1723-1790), Investigación } \\
\text { sobre la naturaleza y causas de la riqueza de las } \\
\text { naciones, } 1776\end{array}$ & Ventaja Absoluta \\
\hline $\begin{array}{l}1.817 \text { David Ricardo, con su ley } \\
\text { de la ventaja comparativa, que ya } \\
\text { subraya cómo los países deberían } \\
\text { competir }\end{array}$ & $\begin{array}{l}\text { véase David Ricardo (1772-1823), Principios } \\
\text { de economia política y tributación, } 1817\end{array}$ & $\begin{array}{l}\text { Ventaja } \\
\text { Comparativa }\end{array}$ \\
\hline $\begin{array}{l}\text { 1.942 Joseph Schumpeter, quien } \\
\text { enfatizó el rol del emprendedor } \\
\text { como un factor de competitividad }\end{array}$ & $\begin{array}{l}\text { véase Joseph Schumpeter (1883-1950), } \\
\text { Capitalismo, socialismo y democracia, } 1942\end{array}$ & $\begin{array}{l}\text { Innovación y Mejora } \\
\text { tecnològica }\end{array}$ \\
\hline $\begin{array}{l}\text { 1.965 Alfred P. Sloan y Peter } \\
\text { Drucker, quienes desarrollaron } \\
\text { con más profundidad el concepto } \\
\text { de administración como un factor } \\
\text { principal para la competitividad }\end{array}$ & $\begin{array}{l}\text { véase Alfred P. Sloan (1875-1965): Mis años } \\
\text { en General Motors; 1963: Peter Drucker, La } \\
\text { era de la discontinuidad, } 1969\end{array}$ & $\begin{array}{l}\text { Administración- } \\
\text { Gerencia }\end{array}$ \\
\hline $\begin{array}{l}\text { 1.990 Finalmente, Michael Porter, } \\
\text { quien ha tratado de integrar todas } \\
\text { estas ideas en un modelo } \\
\text { sistemático llamado el diamante } \\
\text { de la ventaja nacional }\end{array}$ & $\begin{array}{l}\text { véase Michael Porter, La ventaja competitiva } \\
\text { de las naciones, } 1990 .\end{array}$ & Ventaja Competitiva \\
\hline
\end{tabular}

Fuente: (Valero, Rodenes, \& Rueda,

Effects of the use of competitiveness as a strategy on exporting companies:Status of the issue, 2018)

Como se puede apreciar, se encuentra que la competitividad tiene sus inicios en la ventaja absoluta de Adam Smith, clásico que reconoce las ventajas que tienen las economías y que son indiscutibles en la actividad comercial, evolucionando a la ventaja comparativa de David Ricardo que refleja condiciones diferentes en términos de relatividad para el intercambio como elemento decisorio, así mismo la innovación y la empresa desde el concepto administrativo, para terminar con la Ventaja competitiva, planteada por Michael Porter. 
Competitividad para la internacionalizacion, frente a los atributos de innovacion en ColombiaCaso Bucaramanga y su Área Metropolitana

Con base en la revisión de la literatura presentada anteriormente, la competitividad es uno de los aspectos fundamentales a la hora de ingresar al mercado internacional. Cuando una empresa considera la inserción internacional vía exportaciones se debe preparar arduamente para lograr dicha inserción.

Autores como: Porter (1.985); Culloch (1996); Rock (2014); González \& Saldivar (2014); Sosa Rodriguez \& Reyes (2014); Avella (2010); Hill (2001); Daniels (2000); Czinkota (2002), entre otros, consideran la competitividad, elemento fundamental para que las empresas se inserten en el mercado global de manera óptima; plantean que la inmersión internacional se realiza paulatinamente: inicialmente en el mercado local, ajustando las condiciones internas que favorezcan la oferta en el mercado nacional y posteriormente ingresando al mercado global, bien sea como exportadores, importadores $\mathrm{y} / \mathrm{o}$ como inversionistas extranjeros.

De acuerdo al planteamiento de Michael Porter en su libro La ventaja Competitiva, considera la Competitividad como el valor que una empresa logra crear para sus clientes y superar el coste de ella. Es decir, la ventaja competitiva se da en costes bajos y diferenciación (Porter, 2002). El autor considera que la herramienta básica con que se diagnostica la Ventaja Competitiva es a través de la Cadena de Valor que divide una empresa en las actividades para diseñar, producir, comercializar y distribuir sus bienes.

Entonces se puede afirmar, que a través de los años el concepto de competitividad frente al intercambio comercial, determina el ingreso de una economía en el mercado internacional. Hoy en día casi no hay empresa (grande o pequeña) que no resienta los efectos de los acontecimientos y la competencia global, ya que la mayoría de las compañías venden sus productos, reciben provisiones de países extranjeros, o ambas cosas y compiten con productos y servicios procedentes del exterior. (Daniels \& Radebaugh, Negocios internacioinales, 2000).

\subsubsection{Competitividad como estrategia}

En este sentido la competitividad como estrategia, permite afirmar que las empresas se transforman en exportadoras, no de la noche a la mañana, sino paso a paso por medio de un proceso de desarrollo de exportación. Diversos motivos para internacionalizarse, elementos administrativos y corporativos de la empresa, la influencia de los agentes del cambio y la capacidad de la empresa de superar las barreras de la internacionalización, conforman el proceso. (Czinkota \& Ronkainen, 2002).

Con las anteriores consideraciones las empresas determinan la estrategia competitiva a través del patrón común de internacionalización conocido como : "Patrón general que se concentra en la reducción del riesgo al mínimo nivel” (Daniels \& Radebaugh, Negocios Internacionales, 2000), la interpretación a dicho patrón se realiza del centro hacia afuera, y 
considera entre otros la Búsqueda pasiva a activa de oportunidades, el manejo externo a interno de las operaciones (entendidas como exportaciones, importaciones y/o Inversión extranjera) y las modalidades de operación limitadas o ampliadas. Un desplazamiento lento de uno de ellos puede permitir la liberación de recursos que hagan posible a su vez una expansión más rápida en otro.

Los anteriores criterios se constituyen en las variables que evalúan las empresas con disposición estratégica, hacia los mercados internacionales vía exportaciones.

\subsection{Exportaciones (internacionalización)}

En la siguiente tabla, se puede revisar algunas apreciaciones frente a los factores determinantes de las exportaciones, entre los que se encuentran también los siguientes 
Competitividad para la internacionalizacion, frente a los atributos de innovacion en ColombiaCaso Bucaramanga y su Área Metropolitana

Tabla 3. Revision Literatura Exportaciones

\begin{tabular}{|c|c|c|}
\hline TEMA & TEMA/AUTOR & APORTACIONES \\
\hline $\begin{array}{l}\text { Five Facts about Value-Added } \\
\text { Exports and Implications for } \\
\text { Macroeconomics and Trade } \\
\text { Research }\end{array}$ & Robert Johnson (Johnson, 2014) & $\begin{array}{l}\text { Las exportaciones con valor agregado } \\
\text { La fabricación de comercio es } \\
\text { relativamente pequeño, y el comercio } \\
\text { de servicios relativamente más grande, } \\
\text { cuando se mide en términos de valor } \\
\text { añadido }\end{array}$ \\
\hline $\begin{array}{l}\text { El desempeño exportador } \\
\text { basado en la creación de } \\
\text { Capital Social a través del uso } \\
\text { de las Tecnologías de la } \\
\text { Información. Un estudio en el } \\
\text { sector del plástico }\end{array}$ & $\begin{array}{l}\text { María José Peláez Cano (Pelaez } \\
\text { Cano \& Rodenes, 2011) }\end{array}$ & $\begin{array}{l}\text { Para ingresar al mercado internacional } \\
\text { se hace a través de la innovación, la } \\
\text { cual es determinante en el proceso de } \\
\text { exportación y es además un indicador } \\
\text { fundamental para medir el desempeño } \\
\text { exportador }\end{array}$ \\
\hline $\begin{array}{l}\text { The Export Boom of the } \\
\text { Mexican Revolution: } \\
\text { Characteristics } \\
\text { Contributing Factors }\end{array}$ & $\begin{array}{l}\text { Sandra Kuntz Ficker ( Kuntz } \\
\text { Ficker, 2004) }\end{array}$ & $\begin{array}{l}\text { Precios, cantidades y estructura como } \\
\text { ubicación geográfica: factores } \\
\text { determinantes para exportaciones }\end{array}$ \\
\hline $\begin{array}{l}\text { Export promotion enhances } \\
\text { firm's quality reputation, } \\
\text { product and service quality } \\
\text { generating sales and profits: A } \\
\text { structural equation modelling } \\
\text { using AMOS }\end{array}$ & $\begin{array}{l}\text { Hamad A.R (Hamad, Kinson, \& } \\
\text { Shy, 2015) }\end{array}$ & $\begin{array}{l}\text { Las variables latentes que han } \\
\text { marcado altamente el potencial de la } \\
\text { calidad de las firmas en el mercado } \\
\text { internacional son mejorar los } \\
\text { productos, proporcionando una mejor } \\
\underline{\text { calidad de servicio y ganando en }} \\
\text { reputación }\end{array}$ \\
\hline $\begin{array}{l}\text { Location factors of export- } \\
\text { platform FDI: Example of } \\
\text { Vietnam }\end{array}$ & $\begin{array}{l}\text { Minda.Alexandre \& Nguyen, Huu } \\
\text { Thanh Tam (Nguyen, \& Minda, } \\
\text { 2012) }\end{array}$ & $\begin{array}{l}\text { Los costos laborales y la la } \\
\text { productividad son determinantes a la } \\
\text { hora de realizar exportaciones }\end{array}$ \\
\hline Negocios & $\begin{array}{l}\text { Ricky W Griffin \& Ronald J } \\
\text { Ebert (Griffin \& Ebert, 1997) }\end{array}$ & $\begin{array}{l}\text { Determinante para las exportaciones } \\
\text { los siguientes aspectos: Diferencias } \\
\text { sociales-Culturales, } \\
\text { económicas, Diferencias políticas y } \\
\text { legales, Diferencias competitivas. Así } \\
\text { mismo la capacidad de la empresa y el } \\
\text { clima de negocios en otras naciones. }\end{array}$ \\
\hline $\begin{array}{l}\text { Globalización. Estrategias para } \\
\text { obtener una ventaja } \\
\text { competitiva global }\end{array}$ & George S. Yip (Yip, 1992) & $\begin{array}{l}\text { Determinar los siguientes aspectos: } \\
\text { Potencial de globalización, grado de } \\
\text { globalización, } \\
\text { organizacional y cambios necesarios. }\end{array}$ \\
\hline
\end{tabular}

Fuente: (Valero, Rodenes, \& Rueda, La internacionalizacion de las empresas exportadoras, Estado de la Cuestion, 2016) 


\section{Formato de modelo}

Luego de la revisión teorica sobre los ejes fundamentales de la competitividad, la internacioalizacio y la innovación, se plantea a continuación, los aspectos que caracterizan el formato del modelo a plantear; para ello, se presenta a continuacion la Tabla No 4, con los valores e indicadores, basados en las investigaciones (Porter, 2002), (FEM \& Schwad, 1971), (Sistema Nacional de Competitividad, Ciencia, Tecnologia e Innovacion, 2016), (Lugones, Gustavo;), (OCDE-EUROSTAT, 2005), (Zapata Rotundo \& Hernandez, 2014). (Miranda \& Toirac, 2010) (Fajinzylber, 2006), (Jaramillo, Hernan; Lugones, Gustavo; Salazar, Mónica;, 2001).

Tabla 4. Formato de modelo

\begin{tabular}{|c|c|c|c|}
\hline Variable & $\begin{array}{c}\text { Sub- } \\
\text { variables }\end{array}$ & Indicadores & Autores \\
\hline \multirow{4}{*}{ 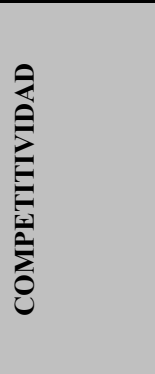 } & \multirow{4}{*}{$\begin{array}{l}z \\
0 \\
0 \\
z \\
0 \\
z \\
\underline{z}\end{array}$} & $\begin{array}{l}\text { Innovacion en } \\
\text { producto/servicios }\end{array}$ & $\begin{array}{l}\text { (BANCO MUNDIAL;, 2016) (FEM \& } \\
\text { Schwad, 1971) (Jaramillo, Hernan; } \\
\text { Lugones, Gustavo; Salazar Mónica; } \\
\text { 2001). (OCDE-EUROSTAT, 2005) }\end{array}$ \\
\hline & & Innovacion en procesos & $\begin{array}{l}\text { (OCDE-EUROSTAT, 2005) (Fajinzylber, } \\
\text { 2006) (Lugones, Gustavo;) }\end{array}$ \\
\hline & & Innovacion organizacional & (OCDE-EUROSTAT, 2005) \\
\hline & & Innovacion en marketing & $\begin{array}{c}\text { ( Consejo Nacional de Politica Economica } \\
\text { y Social, 2006) (CONSEJO PRIVADO } \\
\text { DE COMPETITIVIDAD, 2015) }\end{array}$ \\
\hline
\end{tabular}

Como se puede observar las variables que permiten alcanzar la competitividad via exportaciones se soportan a traves de la Innovación, la cual se mide en: productos/Servicios, procesos, empresa y marketing, tramitadas por la compañía. Igualmente se relacionan los diferentes autores que soportan el formato inicial del modelo.

\section{Metodologia de comprobación}

Bucaramanga y su área metropolitana AMB, es la plataforma del departamento de Santander y se constituye en la base de la internacionalización de su economía, la cual se encuentra conformada por los municipios de Floridablanca, Piedecuesta, Girón y Bucaramanga. De acuerdo al primer informe trimestral de 2016 generado por la Cámara de comercio de Bucaramanga, solo 161 empresas, realizaron exportaciones a 74 países 
Competitividad para la internacionalizacion, frente a los atributos de innovacion en ColombiaCaso Bucaramanga y su Área Metropolitana

destino, con 333 posiciones arancelarias; en dicho informe se encuentra también que el aporte de Santander a las exportaciones del país representa un $2.6 \%$ y las exportaciones excluyendo el petróleo y sus derivados aportaron un 1.4\% (Cámara de Comercio de Bucaramanga, 2016).

De las 161 empresas reportadas en el centro de documentacion de la Cámara de Comercio de Bucaramanga y a través de un cuestionario online, solo 63 emrpesas, dieron respuesta a las preguntas tipo liket que permitieran medir la influencia de la innovacionn en la competitividad de las empresas exportadoras, via inserción internacional. El presente documento se concentra en medir a traves del Coeficiente de Alfa de Cronbach si son pertinente las afirmaciones allí realizadas respecto a la capacidad de innovación en el trabajo, inmerso en las empresas que aportan a la competitividad, como requerimiento para las exportaciones.

\subsection{Innovación en el trabajo}

Dentro del analisis y particularmente la innovación, se encuentra la innovación organizacional que se refiere a la aplicación de nuevos métodos organizativos, que pueden generar cambios en las prácticas empresariales, en la organización del trabajo y en las relaciones de la empresa. Al respecto se busca identificar el comportamiento de las 63 empresas exportadoras de Bucaramanga y su Area Metropolitana que participaron y generaron el nivel de respuestas alcanzadas.

Tabla 5. Organización en el trabajo-capacidad de innovación

\begin{tabular}{|c|c|c|c|c|c|}
\hline Capacidad para innovar en el trabajo de los colaboradores & 1 & 2 & 3 & 4 & 5 \\
\hline $\begin{array}{l}\text { En su empresa se aplican procedimientos formales para evaluar el grado de } \\
\text { riesgo de Proyectos innovadores (Ejem.: métricas, estadísticos de control, } \\
\text { metodologías específicas...) }\end{array}$ & & & & & \\
\hline La empresa envía a los empleados a cursos de formación especializados & & & & & \\
\hline Su empresa fomenta el trabajo en equipo para generar nuevas ideas & & & & & \\
\hline $\begin{array}{l}\text { Como parte del proceso de generación de proyectos innovadores, en la empresa } \\
\text { se practican actividades que fomentan el pensamiento creativo (Ejem. lluvia de } \\
\text { ideas) }\end{array}$ & & & & & \\
\hline En su empresa se realizan proyectos multidisciplinarios & & & & & \\
\hline Al personal se le recompensa por la realización de actividades de innovación & & & & & \\
\hline $\begin{array}{l}\text { El proceso de selección de personal asegura la contratación de personal que } \\
\text { traerá consigo nuevas habilidades e ideas para la empresa }\end{array}$ & & & & & \\
\hline $\begin{array}{l}\text { En su empresa regularmente se consulta información sobre la competencia } \\
\text { (benchmarking) }\end{array}$ & & & & & \\
\hline $\begin{array}{l}\text { Su empresa cuenta con una Intranet para utilizar el conocimiento generado por } \\
\text { la organización }\end{array}$ & & & & & \\
\hline $\begin{array}{l}\text { Se utilizan nuevas tecnologías de la información y la comunicación (TIC) para } \\
\text { gestionar el conocimiento }\end{array}$ & & & & & \\
\hline
\end{tabular}

Fuente: Elaboraciòn propia 
Como se puede observar, se plantean 10 preguntas, que permitan identificar el comportamiento de la innovación en el trabajo, inmerso en las empresas encuestadas via exportaciones.

Al evaluar la consistencia interna de la pregunta relacionadas con capacidad para innovar en el trabajo, se observó que las 10 preguntas (con escala de respuesta en formato Likert) tienen un coeficiente de Alfa de Cronbach de 0.932, que refleja una alta consistencia o relación entre las preguntas (ver tabla 6), que garantiza la posibilidad de construir un indicador numérico, posterior al presente analisis.

Tabla 6. Análisis de fiabilidad de los indicadores de capacidad para innovar en el trabajo

\begin{tabular}{l|l}
\multicolumn{2}{l}{ Estadísticas de fiabilidad } \\
Alfa de Cronbach & $\mathrm{N}$ de elementos \\
& \\
\hline 932 & 10 \\
\hline
\end{tabular}

De igual forma, se ilustra en la siguiente tabla, el compartamiento del coeficiente de Alfa de Cronbach para cada una de las preguntas con el fin de corroborar la consistencia de las mismas. Dicha información se encuentra en la siguiente tabla:

Tabla 7. Anàlisis de datos para la Innovaciòn en el trabajo

\begin{tabular}{|l|l|l|l|l|}
\hline Subvariables & $\begin{array}{l}\text { Media de } \\
\text { escala }\end{array}$ & $\begin{array}{l}\text { Varianza } \\
\text { de escala }\end{array}$ & $\begin{array}{l}\text { Correlació } \\
\text { n total }\end{array}$ & $\begin{array}{l}\text { Alfa de } \\
\text { cronbach }\end{array}$ \\
\hline 1_PROCED_RIESGO_PROY & 32,74 & 61,142 &, 677 &, 928 \\
\hline 2_FORMA_CURSO_ESP & 32,83 & 58,356 &, 718 &, 925 \\
\hline 3_FOMENTO_TRABA_EQUI & 32,66 & 60,405 &, 686 &, 927 \\
\hline 4_PENSAMI_CREATI & 32,86 & 57,735 &, 770 &, 923 \\
\hline 5_PROY_MULTIDISC & 32,90 & 57,147 &, 756 &, 923 \\
\hline 6_RECOMP_INNOVA & 32,79 & 57,746 &, 790 &, 922 \\
\hline 7_SELECC_PERS_INNOVA & 32,81 & 57,946 &, 734 &, 925 \\
\hline 8_CONSL_COMPET_BENCH & 32,84 & 57,256 &, 790 &, 922 \\
\hline 9_INTRANET_CONOC & 32,78 & 57,616 &, 722 &, 925 \\
\hline 10_NUEVAS_TIC_GESTCONO & 32,67 & 58,294 &, 682 &, 928 \\
\hline
\end{tabular}


Competitividad para la internacionalizacion, frente a los atributos de innovacion en ColombiaCaso Bucaramanga y su Área Metropolitana

Como se puede apreciar, todas las respuestas superan el análisis de fiabilidad según el Alfha de Cronbach que debe ser superior o igual a 0,6 asi como la media, la varianza y la correlacion para cada una de ellas. Lo anterior muestra un alto nivel de consistencia entre las mismas. Por tanto se puede afirmar que el formato modelo respecto a las preguntas y el comportamiento de las mismas según el indicador (alfa de cronbach) es pertinente.

\section{Conclusiones, aportaciones y futuras lineas de trabajo}

Se reconoce la importancia de la Innovacion como insumo fundamental inmerso en las empresas.

Las empresas deben trabajar para lograr la competitividad, como requisito indispensable hacia el ingreso en mercados internacionales.

La inserción de las empresas al mercado global, implica que las mismas estudien y evalúen permanentemente sus procesos técnicos y humanos.

La competitividad, esta íntimamente relacionada con la innovación, y esta a su vez con el compromiso humano, resultado de los lineamientos de la empresa.

La innovacion surge de cada uno de los colaboradores de la empresa quienes aportan diariamente de acuerdo al nivel de motivación que cada uno de ellos tenga como resultado de las disposicones de la compañía.

Para Bucaramanga y su Area Metropolitana existe un buen nivel de correspondencia a las preguntas efectuadas a las empresas exportadoras objeto de aplicación.

Existe una alta correspondencia (alfa de Cronbach) en elementos de innovación relacionados con la iniciativa personal y los espacios generados por la misma empresa.

El comportamiento y el resultado de las preguntas respalda la pertiencia del formato modelo, el cual se constituye en un buen elemento de comprobación hacia futuras comprobaciones.

\section{Referencias}

Arge, G., Dajer Plata, R., \& Alvarez, D. (s.f.). Diseño e implementación de un mapa de ordenamiento competitivo regional soportado en tecnología internet para asistir procesos de inversión en el departamento de Cordoba-Colombia. En ASCOLFA (Ed.), Conferencia Nacional ASCOLFA (pág. 183 a225). ASCOLFA.

Consejo Nacional de Politica Economica y Social. (2006). INSTITUCIONALIDAD $Y$ PRINCIPIOS RECTORES DE POLÍTICA PARA LA COMPETITIVIDAD Y PRODUCTIVIDAD . Bogota. 
BANCO MUNDIAL. (23 de MARZO de 2016). Obtenido de http://www.bancomundial.org/

BANCO MUNDIAL; (23 de MARZO de 2016). Obtenido de http://www.bancomundial.org/

Cámara de Comercio de Bucaramanga. (marzo de 2016). http://www.camaradirecta.com/temas/documentos\%20pdf/exporta2016/exportacio nes_marzo_2016.pdf.

Comision regional de Competitividad. (25 de marzo de 2016). Santander Competitivo. Obtenido de Santander Competitivo- Comision Regional de Competitividad: http://santandercompetitivo.org/secciones-14-s/quienes-somos.htm

CONSEJO PRIVADO DE COMPETITIVIDAD. (2015). Indice Departamental de Competitividad 2015.

Czinkota, M. R., \& Ronkainen, I. A. (2002). Marketing Internacional. En M. R. Czinkota, \& I. A. Ronkainen, Marketing Internacional- sexta edicion (págs. 224-241). México: Pearson Educación.

Daniels, J. D., \& Radebaugh, L. H. (2000). Negocios internacioinales. En J. D. Daniels, \& L. H. Radebaugh, Negocios Internacionales Octava edicion (págs. 8-9). Mexico: Pearson Education.

Daniels, J. D., \& Radebaugh, L. H. (2000). Negocios Internacionales. En J. D. Daniels, \& L. H. Radebaugh, Negocios Internacionales-octava edicion (págs. 23-28). Mexico: Pearson Educacion.

Fajinzylber, F. (2006). Una Vision Renovadora del Desarrollo de America Latina. Chile: CEPAL.

FEM, \& Schwad, K. (Enero de 1971). Foro Económico Mundial FEM. Recuperado el 2016, de http:/www.weforum.org/world-economic-forum: http://www.weforum.org/world-economic-forum

Garelli, S. (2006). "The competitiveness of nations: the fundamentals", imd World Competitivenes Yearbook 2006, 2006 [en linea] http://www.imd.ch/documents/wcc/content/Fundamentals.

Griffin, R. W., \& Ebert, R. J. (1997). Negocios. Mexico: Prentice Hall.

Hamad, A. R., Kinson, N. G., \& Shy, C. (2015). Export promotion enhances firm's quality reputation, product and service quality generating sales and profits: A structural equation modelling using AMOS (Conference Paper). Proceedings of the 25th International Business Information Management Association Conference - 
Competitividad para la internacionalizacion, frente a los atributos de innovacion en Colombia-

Caso Bucaramanga y su Área Metropolitana

Innovation Vision 2020: From Regional Development Sustainability to Global Economic Growth, IBIMA , 146-159.

Jaramillo, Hernan; Lugones, Gustavo; Salazar Mónica;. (2001). Manual de Bogota : Normalización de Indicadores de Innovación Tecnológica en América Latina y el Caribe.

Jaramillo, Hernan; Lugones, Gustavo; Salazar, Mónica;. (2001). Manual de Bogota : Normalización de Indicadores de Innovación Tecnológica en América Latina y el Caribe.

Johnson, R. C. (2014). Five Facts about Value-Added Exports and Implications for Macroeconomics and Trade Research. The Journal of Economic Perspectives Volumen 28 No 2, 119-142.

Kuntz Ficker, S. (2004). The Export Boom of the Mexican Revolution: Characteristics and Contributing Factors . Journal of Latin American Studies Vol 36 No 2, 267-296.

Lugones, Gustavo;. (s.f.). Módulo de capacitación para la recolección y el análisis de indicadores de innovación. Redes Banco Interamericano de Desarrollo BID.

Miranda , J., \& Toirac, L. (2010). Indicadores de Productividad para la Industria Dominicana. Ciencia y Sociedad, 235-290.

Nguyen,, H., \& Minda, A. (2012). Location factors of wxport-plataform FDI: Example of Vietnam. Revew Economic Volumen 63, 69-91.

OCDE-EUROSTAT. (2005). Manual de Oslo. Obtenido de Manual de Oslo.

Pelaez Cano, M. J., \& Rodenes, M. (17 de 06 de 2011). El desempeño exportador basado en la creacion de Capital Social a traves del uso de las Tecnologias de la Informacion. Un estudio en el sector del plástico . Valencia, España.

Porter, M. E. (2002). Ventaja Competitiva- Creación y sostenimiento de un desempeño superior. Mexico: CECSA.

Sanchez, F., \& Acosta, P. (2001). Proyecto Andino de Competitividad : Proyecto Indicadores de Competitividad Colombia. Bogota: CEDE Universidad de los Andes.

Simon Gaviria Muñoz. (2016). Una apuesta en la que todos ganan. Ciencia Tecnologia e Inovacion 2016- Colciencias-Semana, 162-164.

Sistema Nacional de Competitividad, Ciencia, Tecnologia e Innovacion. (23 de marzo de 2016).

Obtenido

de 
http://www.colombiacompetitiva.gov.co/sncei/Paginas/indicadoresnacionales.aspx

Universidad del Rosario. (29 de 09 de 2009). http://repository.urosario.edu.co/handle/10336/1462. (Universidad del Rosario, Editor) Recuperado el 07 de 01 de 2016, de http://repository.urosario.edu.co/handle/10336/1462:

http://biblioteca.versila.com/15818764

Valero, G. M., Rodenes, M., \& Rueda, G. E. (2016). La internacionalizacion de las empresas exportadoras, Estado de la Cuestion. Lebret, 127-147.

Valero, G. M., Rodenes, M., \& Rueda, G. E. (2018). Effects of the use of competitiveness as a strategy on exporting companies:Status of the issue. Equidad y Desarrollo, $173-194$.

Valero, G. M., Rodenes, M., \& Rueda, G. E. (2018). Effects of of the use of competitiveness as a strategy on exporting companies: Status of the issue. Equidad y Desarrollo, $173-194$.

Yip, G. S. (1992). Globalizacion. Estrategias para obtener una ventaja competitiva global. Bogotá: Norma.

Zapata Rotundo, G. J., \& Hernandez, A. (2014). Origen de los recursos y ventajas competitivas de las organizaciones: reflexiones teóricas. Utopia y Praxis Latinoamericana, 735-759. 